\title{
Surgical Treatment of Unruptured Brain AVMs: Short- and Long-Term Results
}

\author{
Shalva Eliava, Vadim Gorozhanin, Oleg Shekhtman, \\ Yuri Pilipenko, and Olga Kuchina
}

\section{Introduction}

Brain arteriovenous malformations (bAVM) are one of the most common congenital vascular brain malformations, which frequently manifests at young age [1]. Besides hemorrhages, patients may exhibit seizures (17-40\%) and chronic headaches $(9.8-46 \%)$ that decrease the quality of life [2]. Growing access to high-quality imaging has increased the number of patients with unruptured bAVM. The annual risk of bAVM hemorrhage reported previously is $1.7-3.1 \%$ [37]. Mortality after rupture reaches $29 \%$ and permanent morbidity occurs in more than one-third of cases [8].

Microsurgery remains the most effective treatment option and is considered a first-line treatment for ruptured bAVMs [9-11]. However, the clinical approach to unruptured AVMs remains under discussion. ARUBA randomized trial and the following SAIVMS showed that conservative management is more beneficial than invasive modalities. Trial results were followed by a wave of criticism from neurosurgeons across the globe. Many specialists emphasized numerous biases that could have affected its results. Further surgical series for unruptured bAVMs published in the "post-Aruba era" mostly overruled ARUBA conclusions. The presented work reflects our experience with unruptured AVM patients operated upon in Burdenko National Research Center for Neurosurgery.

\section{Materials and Methods}

The retrospective study included 160 adult patients (>18 years old) with unruptured bAVMs admitted for microsurgery in 2009-2017. Of these, 93 (58.1\%) were males and

\footnotetext{
S. Eliava $(\bowtie) \cdot$ V. Gorozhanin · O. Shekhtman · Y. Pilipenko O. Kuchina

National Medical Research Center "Burdenko Neurosurgical Institute", Moscow, Russia

e-mail: eliava@nsi.ru; vgorozhanin@nsi.ru; oshekhtman@nsi.ru; ypilipenko@nsi.r; okuchina@nsi.ru
}

$67(41.9 \%)$ were females. Mean age was $33.4 \pm 10$ years (range 18-67 years). No signs of hemorrhage were found on brain MRI or CT in all cases. Seizures were the most common clinical sign, found in 99 patients $(61.9 \%)$, and 49 patients $(30.6 \%)$ had chronic headaches, four $(2.5 \%)$ had transient ischemic symptoms, and in eight (5\%) the AVMs were asymptomatic. Most patients with seizures (86\%) had generalized seizures and only three patients had secondarily generalized seizures.

The decision for surgical treatment was based on SpetzlerMartin score (Grade I-III), clinical signs (permanent decrease of quality of life), AVM location (eloquence, supply, angiomatosis, etc.), and age (in terms of cumulative hemorrhage risk). In addition, a small group of patients with AVM Spetzler-Martin Grade IV (high risk) who had severe, disabling symptoms (pharmacoresistant seizures, persistent headache ineffectively controlled with medications) was included. Patient data were extracted from the electronic medical records (e-med). Before and after surgery patients were examined by a neurologist and ophthalmologist and electroencephalography (EEG) was done for seizure patients. AVM morphology was evaluated with cerebral angiography (CA) or spiral CT angiography (CTA). All patients had a control angiography postoperatively. Outcomes were assessed with modified Rankin scale (mRS) at discharge (10-14 days). Follow-up exams were carried in the outpatient department or over the phone.

\section{Results}

The distribution of patients by Spetzler-Martin score, AVM size, and location is presented in Table 1. Mean AVM size was $3.3 \pm 1.07 \mathrm{~cm}(1.5-10 \mathrm{~cm})$. Patients with AVM in the temporal $(72 \%)$ and frontal $(70 \%)$ lobes had a higher tendency for seizures. Of all patients with occipital AVMs, 13 manifested with headaches and five had photopsias (all AVMs were $<4 \mathrm{~cm}$ ). In six patients with occipital AVM, 
Table 1 Distribution of AVMs by Spetzler-Martin score, size, and location

Spetzler-Martin score and AVM size, $n(\%)$

\begin{tabular}{l|l|l|l}
\hline I & $18(11.3 \%)$ & Small $(1-3 \mathrm{~cm})$ & $55(34.4 \%)$ \\
\hline II & $71(44.4 \%)$ & Medium $(3.5-5.5 \mathrm{~cm})$ & $87(54.4 \%)$ \\
\hline III & $60(37.5 \%)$ & Large $(\geq 6 \mathrm{~cm})$ & $18(11.2 \%)$ \\
\hline IV & $11(6.8 \%)$ & & \\
\hline
\end{tabular}

\begin{tabular}{l|l|l|l}
\hline Location, $n(\%)$ & \\
\hline Frontal lobe & $60(37.5 \%)$ & Parietal lobe & $10(6.2 \%)$ \\
\hline Temporal lobe & $42(26.3 \%)$ & Occipitotemporal & $8(5 \%)$ \\
\hline Occipital lobe & $22(13.8 \%)$ & Cerebellum & $4(2.5 \%)$ \\
\hline Occipitoparietal & $13(8.1 \%)$ & Temporoparietal & $1(0.6 \%)$
\end{tabular}

visual field defects that could not be associated with hemorrhages were found before surgery (homonymous hemianopia-five, quadrant hemianopia-one). These defects may have been congenital or due to asymptomatic ischemic changes).

Surgery. Microsurgical resection of AVM was performed in $128(80 \%)$ patients, resection with preoperative embolization in $31(19.4 \%)$. In one case, surgery was limited to AVM afferent clipping due to critical M-response decrease. In three cases simultaneous epilepsy resection using ECoG monitoring (amygdalohippocampectomy or temporal lobectomy) was performed. AVMs were located in the mediobasal temporal lobe in two patients and in the hippocampus in one patient.

In 33 cases $(20.6 \%)$ minor hemorrhage signs ("silent" hemorrhage) were identified during surgery. Commonly, these included local hemosiderin deposits limited to the one or two sides of the lesions. No typical parenchymal hemorrhages in the form of hematomas residuals or posthemorrhagic cysts were identified. Results of surgical treatment are summarized in Fig. 1. Favorable outcomes (mRS $=0-2)$ at discharge were achieved in 149 patients (93.1\%), and satisfactory outcomes (mRS-3) in $9(5.6 \%)$ patients. Two patients died $(1.25 \%)$, one due to the severe air embolism, the other from the complicated pre-surgical embolization (glue migration).

The long-term treatment outcomes were evaluated in 97 $(60.6 \%)$ patients. Mean duration of follow-up was 59.3 months (13-108 months). At follow-up, the number of patients with excellent outcomes ( $\mathrm{mRS}=0-1)$ increased to $80.4 \%$, and the total number of favorable outcomes $(\mathrm{mRS}=0-2)$ reached $94.8 \%$. Two $(2 \%)$ patients died of causes unrelated to surgery.

The long-term outcomes were followed in 59 of 99 patients $(55.6 \%)$ with seizures. Engel class I was achieved in 50 patients $(84.8 \%$ ) (complete regression-64.4\%, improvement-30.5\%); Engel class IIA was achieved in two (3.4\%), Engel class IIIA in four (6.8\%), and Engel class IV in three patients $(5.1 \%)$. The headache intensity over the time was evaluated in 65 of 87 patients (74.7\%). Headache intensity

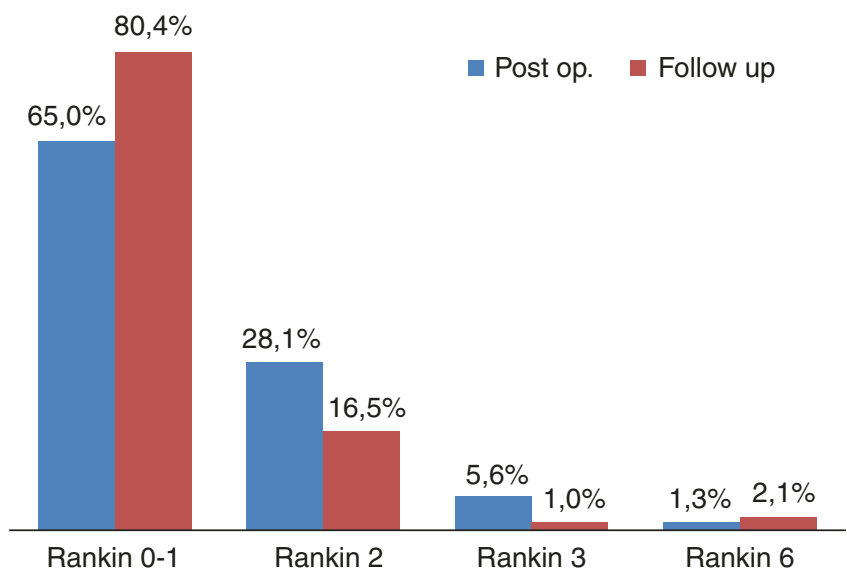

Fig. 1 Surgical outcomes for unruptured bAVM at discharge and at follow-up

decreased after surgery in 41 patients $(63 \%)$, frequency decreased in two (3.1\%), headache worsened in twelve $(18.5 \%)$, and remained unchanged in ten $(15.4 \%)$.

Complications. Hemianopia was the most common postsurgical neurological deficit (in some a combination with central scotomas were observed) and was reported in 55 cases $(64.7 \%$ ) (homonymous hemianopia-39, quadrant hemianopia-16). Visual field defects occurred in $100 \%$ of AVMs found in the mediobasal temporal lobe $(n=6)$ and in the occipital-temporal area $(n=8)$; in $92.3 \%$ of AVMs found in the occipitoparietal area $(n=12)$, and in $86.3 \%(n=19)$ of those found in the occipital lobe AVMs. In $13.7 \%$ of patients with occipital AVM, visual fields after surgery remained unchanged. Postoperative visual field defects were followed in 22 of 55 patients (40\%) (mean follow-up duration was 61.7 months), including ophthalmologist examination and perimetry. Complete recovery of visual defects was reported in six patients (27\%), partial recovery in eight (36\%), vision was unchanged in seven (32\%), and in one patient visual field defects worsened.

Other postoperative neurological complications included motor deficit in nine patients, aphasia in six patients, cognitive disorders including behavior changes in six patients, and cerebellar or vestibular dysfunctions in two patients. In two cases hemorrhages occurred after AVM embolization. Hematomas and AVMs were successfully removed with a good outcome in both cases.

\section{Discussion}

Generally, surgical treatment for unruptured bAVMs is recommended for young patients with high life expectancy (in this study, $77 \%$ of patients were aged $<40$ years) and low surgical risks (S-M I-III). A separate category, not analyzed in our work, is young women planning a pregnancy. As 
K. Fukuda showed, the first AVM hemorrhage may be fatal to $28 \%$ of women and $14 \%$ of fetuses, as well as causing early miscarriage $[12,13]$. Although the influence of labor on the AVM rupture is still under discussion, some studies have identified a higher risk of rupture compared to the general population $[13,14]$.

In most patients, symptomatic epilepsy was an indication for surgery. According to our data, favorable outcomes were achieved in $84.8 \%$ of patients (Engel class I) with complete seizures relieved (Engel class IA) in 64\%. AVM resection does not ensure full seizure resolution; therefore, we recommend continuation of anticonvulsants for 6-12 months. According to the published reports, AVM resection is a quite effective treatment and results in seizure control (Engel class I) in $52-100 \%$ of patients [15-18].

Correlation between bAVMs and chronic headaches is unclear and remains a subject of further investigation. Of all bAVM patients, those with headaches comprise a significant percentage, but it is difficult to establish relevant association between headache and AVM [19-21]. According to the published reports, surgical resection of AVM led to headache intensity decrease or complete resolution in $33-83 \%$ of patients [22-24]. In our series, $66 \%$ of patients demonstrated various degrees of improvement.

Visual cortex and tracts are the most sensitive areas in terms of postoperative neurological defects. High risk factors for visual compromise are AVM size $>3 \mathrm{~cm}$ and proximity to temporal, temporo-occipital or parieto-occipital cortex. It is also important to consider AVM angiomatosis that may require extended resection that exceeds initially planned boundaries. Although the group with follow-up ophthalmological examination was small $(n=22)$, field defects improvement was confirmed in more than half $(63 \%)$ of the patients. Further study with more patients is obviously needed.

Endovascular embolization reduces the AVM volume and thus facilitates subsequent resection. To minimize hemorrhage risks between surgical steps it is an established practice in our department to focus an endovascular session on occlusion of hemodynamic aneurysms, embolization of deep afferents and AVM parts that are difficult for excision or excess during open surgery. A coordinated schedule of patient treatment and transportation may reduce time between both stages. In future, hybrid operating rooms may become a solution to such cases.

Functional outcomes in our study are consistent with the published data confirming that surgery for "low-grade" AVMs is reasonably safe and not associated with major neurological complications. Indications for surgery for unruptured bAVMs Spetzler-Martin Grade III-IV are individual and justified only when better quality of life is expected (e.g., improvement of the symptomatic epilepsy) rather than hemorrhage prevention. Patients with AVM Spetzler-Martin
Grade V should be managed conservatively regardless of the clinical signs. The effectiveness of the chronic headaches (migraine) treatment in patients with AVM is questionable and further studies are warranted.

Acknowledgments We wish to express our sincere gratitude to Dr. Elena Okisheva for editing and translation assistance.

Conflict of interest. Authors declare that they have no conflict of interest.

\section{References}

1. Tong $\mathrm{X}$, Wu J, Lin F, Cao Y, Zhao Y, Ning B, Zhao B, Wang L, Zhang S, Wang S, Zhao J (2016) The effect of age, sex, and lesion location on initial presentation in patients with brain arteriovenous malformations. World Neurosurg 87:598-606

2. Hoh BL, Chapman PH, Loeffler JS, Carter BS, Ogilvy CS (2002) Results of multimodality treatment for 141 patients with brain arteriovenous malformations and seizures: factors associated with seizure incidence and seizure outcomes. Neurosurgery 51:303-309

3. Brown RD Jr, Wiebers DO, Forbes GS (1990) Unruptured intracranial aneurysms and arteriovenous malformations: frequency of intracranial hemorrhage and relationship of lesions. J Neurosurg 73:859-863

4. Hernesniemi JA, Dashti R, Juvela S, Väärt K, Niemelä M, Laakso A (2008) Natural history of brain arteriovenous malformations: a long-term follow-up study of risk of hemorrhage in 238 patients. Neurosurgery 63(5):823-829. discussion 829-31

5. Ondra SL, Troupp H, George ED, Schwab K (1990) The natural history of symptomatic arteriovenous malformations of the brain: a 24-year follow-up assessment. J Neurosurg 73(3):387-391

6. da Costa L, Wallace MC, Ter Brugge KG, O'Kelly C, Willinsky RA, Tymianski M (2009) The natural history and predictive features of hemorrhage from brain arteriovenous malformations. Stroke 40(1):100-105

7. Yamada S, Takagi Y, Nozaki K, Kikuta K, Hashimoto N (2007) Risk factors for subsequent hemorrhage in patients with cerebral arteriovenous malformations. J Neurosurg 107(5):965-972

8. Gross BA, Du R (2012) Surgical and radiosurgical results of the treatment of cerebral arteriovenous malformations. J Clin Neurosci 19:1001-1004

9. Gross BA, Du R (2012) Rate of rebleeding of arteriovenous malformations in the first year after rupture. J Clin Neurosci 19:1087-1088

10. Heros RC, Korosue K, Diebold PM (1990) Surgical excision of cerebral arteriovenous malformations: late results. Neurosurgery 26:570-578

11. Pikus HJ, Beach ML, Harbaugh RE (1998) Microsurgical treatment of arteriovenous malformations: analysis and comparison with stereotactic radiosurgery. J Neurosurg 88:641e646

12. Dias MS, Sekhar LN (1990) Intracranial haemorrhage from aneurysms and arteriovenous malformations during pregnancy and the puerperium. Neurosurgery 27:444-446

13. Fukuda K, Hamano E, Nakajima N, Katsuragi S, Ikeda T, Takahashi J (2013) Pregnancy and delivery management in patients with cerebral arteriovenous malformation: a single-center experience. Neurol Med Chir (Tokyo) 53:565-570

14. Porras JL, Yang W, Philadelphia E, Law J, Garzon-Muvdi T, Caplan JM, Colby GP, Coon AL, Tamargo RJ, Huang J (2017) Hemorrhage risk of brain arteriovenous malformations during pregnancy and puerperium in a north American cohort. Stroke 48(6):1507-1513

15. Hyun SJ, Kong DS, Lee JI, Kim JS, Hong SC (2012) Cerebral arteriovenous malformations and seizures: differential impact on the 
time to seizure-free state according to the treatment modalities. Acta Neurochir 154:1003-1010

16. Josephson CB, Bhattacharya JJ, Counsell CE, Papanastassiou V, Ritchie V, Roberts R, Sellar R, Warlow CP, Al-Shahi Salman R, Scottish Audit of Intracranial Vascular Malformations (SAIVMs) Steering Committee and Collaborators (2012) Seizure risk with AVM treatment or conservative management: prospective, population based study. Neurology 79(6):500-507

17. Wang JY, Yang W, Ye X, Rigamonti D, Coon AL, Tamargo RJ, Huang J (2013) Impact on seizure control of surgical resection or radiosurgery for cerebral arteriovenous malformations. Neurosurgery 73:648-655

18. Rohn B, Hänggi D, Etminan N, Turowski B, Steiger HJ (2017) Relief of epilepsy and headache and quality of life after microsurgical treatment of unruptured brain AVM-audit of a single-center series and comprehensive review of the literature. Neurosurg Rev 40(1):59-65

19. Headache Classification Committee of the International Headache Society (2013) The international classification of headache disorders, 3rd edition (beta version). Cephalalgia 33:629-808
20. ApSimon HT, Reef H, Phadke RV, Popovic EA (2002) A populationbased study of brain arteriovenous malformation: long-term treatment outcomes. Stroke 33:2794-2800

21. Brown RD Jr, Wiebers DO, Torner JC, O'Fallon WN (1996) Incidence and prevalence of intracranial vascular malformations in Olmsted County, Minnesota, 1965 to 1992. Neurology 46:949-952

22. Steiger HJ, Etminan N, Hänggi D (2014) Epilepsy and headache after resection of cerebral arteriovenous malformations. Acta Neurochir Suppl 119:113-115

23. Dehdashti AR, Thines L, Willinsky RA, ter Brugge KG, Schwartz ML, Tymianski M, Wallace MC (2010) Multidisciplinary care of occipital arteriovenous malformations: effect on nonhemorrhagic headache, vision, and outcome in a series of 135 patients. Clinical article. J Neurosurg 113:742-748

24. Lundqvist C, Wikholm G, Svendsen P (1996) Embolization of cerebral arteriovenous malformations: part II-aspects of complications and late outcome. Neurosurgery 39:460-467

Open Access This chapter is licensed under the terms of the Creative Commons Attribution 4.0 International License (http://creativecommons. org/licenses/by/4.0/), which permits use, sharing, adaptation, distribution and reproduction in any medium or format, as long as you give appropriate credit to the original author(s) and the source, provide a link to the Creative Commons license and indicate if changes were made.

The images or other third party material in this chapter are included in the chapter's Creative Commons license, unless indicated otherwise in a credit line to the material. If material is not included in the chapter's Creative Commons license and your intended use is not permitted by statutory regulation or exceeds the permitted use, you will need to obtain permission directly from the copyright holder. 